\title{
Análise do comportamento de corpos de prova cilíndricos com duas partes aderidas por epóxi em concretos com diferentes resistências e inclinações
}

\author{
Analysis of behavior of cylindrical specimens \\ with two pieces bonded by epoxy in \\ concrete with different strengths \\ and inclinations
}

Iporan de Figueiredo Guerrante ${ }^{1}$, Emil de Souza Sánchez Filho², Luiz Antonio Vieira Carneiro ${ }^{3}$

\footnotetext{
${ }^{1}$ Universidade Federal Fluminense - UFF - Rua Passo da Pátria, 156, $3^{\circ}$ andar, São Domingos, Niterói, RJ. e-mail: iporan@terra.com.br

${ }^{2}$ Universidade Federal Fluminense - UFF - Rua Passo da Pátria, 156, $3^{\circ}$ andar, São Domingos, Niterói, RJ. e-mail: emilsanchez@uol.com.br

${ }^{3}$ Instituto Militar de Engenharia - Praça General Tibúrcio, 80, Praia Vermelha, Urca, Rio de Janeiro, RJ e-mail: carneiro@ime.eb.br
}

\section{RESUMO}

Este estudo teórico-experimental visa analisar o estado de tensão atuante nas juntas de aderência de corpos de prova cilíndricos, aderidos com epóxi e com concretos de diferentes resistências (21,6 MPa, 30,0 MPa, $72,8 \mathrm{MPa}$ e $86,6 \mathrm{MPa}$ ) com inclinações de $30^{\circ}, 45^{\circ}$ e $60^{\circ}$ e a rugosidade média das peças de concreto. A rugosidade de cada peça foi analisada por meio de microscópio digital. Foram realizados noventa e três ensaios à compressão, e determinados os valores médios para tensão de ruptura, tensão normal ao plano da junta e tensão de aderência. Para a análise da rugosidade foram tiradas 744 fotografias distribuídas entre as classes de concreto pesquisadas. Os resultados são analisados mostrando o comportamento das tensões nas juntas de colagem, a correlação entre os tipos de concreto e a rugosidade média de cada classe de concreto.

Palavras-chave: concreto, concreto de alta resistência, resina epóxi, rugosidade, estruturas.

\section{ABSTRACT}

This theoretical and experimental study aims to analyze the stress state acting at the bond joints of cylindrical specimens, mounted with concrete of different strengths (21,6 $\mathrm{MPa}, 30,0 \mathrm{MPa}, 72,8 \mathrm{MPa}$ and 86,6 MPa) with inclination of $30^{\circ}, 45^{\circ}$ and $60^{\circ}$ and the average roughness of the concrete pieces. The roughness of each specimen was analyzed using a digital microscope. Ninety-three compressive tests were carried out, and determined the average values for stress state. For the analysis of roughness were taken seven hundred fortyfour photographs distributed among the investigated concrete classes. The results are analyzed and the behavior of the stresses in the joints bonding, the correlation between the types of concrete and the average roughness of each concrete class are presented herein.

Keywords: concrete, high strength concrete, epoxy resin, roughness, structures.

\section{INTRODUÇÃO}

A análise dos elementos pré-moldados de concreto, quando da sua união com as peças concretadas in loco, é complexa devido aos diversos parâmetros a serem considerados. Vários desses parâmetros ainda não foram adequadamente estudados e carecem de validação experimental. A filosofia de dimensionamento das normas e códigos internacionais apresentam formulações deficientes para essa análise, o que leva a dúvidas quanto 
ao dimensionamento estrutural. Este artigo apresenta uma concisa e seletiva análise de um modelo para representar a rugosidade de superfícies de concreto a serem unidas. O estudo de juntas e conexões de concreto são fundamentais para as estruturas pré-moldadas, tanto na fase de projeto como no reparo e reabilitação de estruturas de pontes. O programa teórico-experimental desenvolvido, aqui apresentado de forma concisa, fornece informaçõessubsídios para o dimensionamento, análise e normalização e visa fornecer subsídios para pesquisas futuras.

\section{SIGNIFICÂNCIA DA PESQUISA}

A utilização de concretos com resistências cada vez mais elevadas em estruturas pré-moldadas leva à necessidade de aprimorar o entendimento do comportamento da junção entre elementos estruturais de concreto. Esse comportamento é dependente da análise das resistências à compressão dos concretos utilizados, da superfície de contato a ser colada, da inclinação da junta e do adesivo utilizado. Neste artigo são analisadas todas essas variáveis de modo a propiciar dados consistentes para a elaboração de um modelo de análise para esse problema. A recente publicação do Model Code 2010 da FIB mostra que esse assunto é atual e carece de mais dados para o seu desenvolvimento.

\section{SOLICITAÇÔES EM JUNTAS DE CONCRETO}

A analogia de atrito de Coulomb fornece resultados que permitem estimar a transferência de tensões cisalhantes ao longo das fissuras de elementos estruturais de concreto e várias contribuições teóricas e experimentais têm aprimorado a representação do comportamento das solicitações em juntas de concreto.

BIRKELAND e BIRKLAND [6] estudaram conexões na construção de concreto pré-moldado. A relevância desse estudo levou a uma série de discussões sobre o tema, uma vez que o processo construtivo de estruturas pré-moldadas necessitava de soluções técnicas rápidas e fundamentadas teoricamente. A hipótese de atrito formulada por esses pesquisadores (Figura 1) admite que as conexões ocorrem entre blocos de concreto similares, existindo um plano de ruptura entre eles.
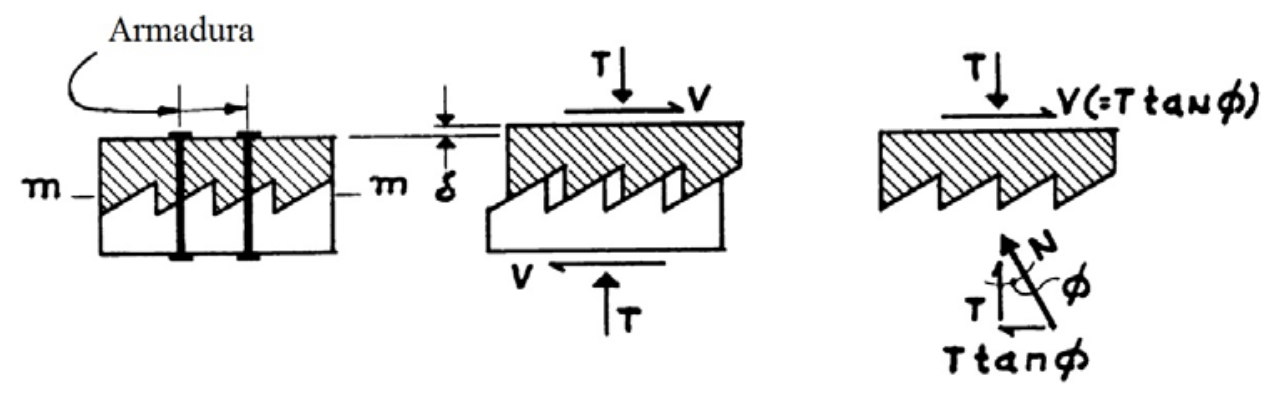

Figura 1: Hipótese de atrito utilizada por BIRKELAND e BIRKLAND [6].

No decorrer das últimas décadas vários pesquisadores têm contribuído para o desenvolvimento teórico-experimental do estudo de juntas e conexões em estruturas de concreto. EIBL et al. [8] utilizando corpos de prova prismáticos desenvolveram pesquisas sobre a adesão entre concretos (Figura 2), adicionando resinas epóxi à argamassa utilizada como ligação entre as peças pré-moldadas de concreto, e concluíram que a introdução do aditivo aumenta a resistência da junção em 2,6 vezes em relação à do concreto normal utilizado. 


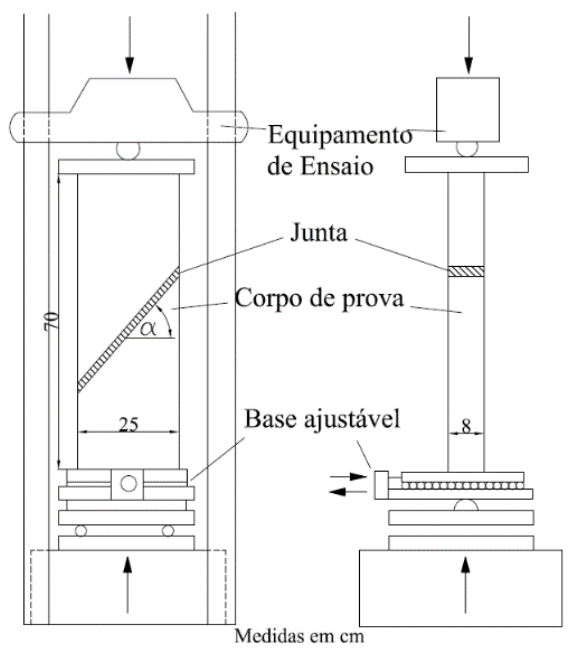

a)

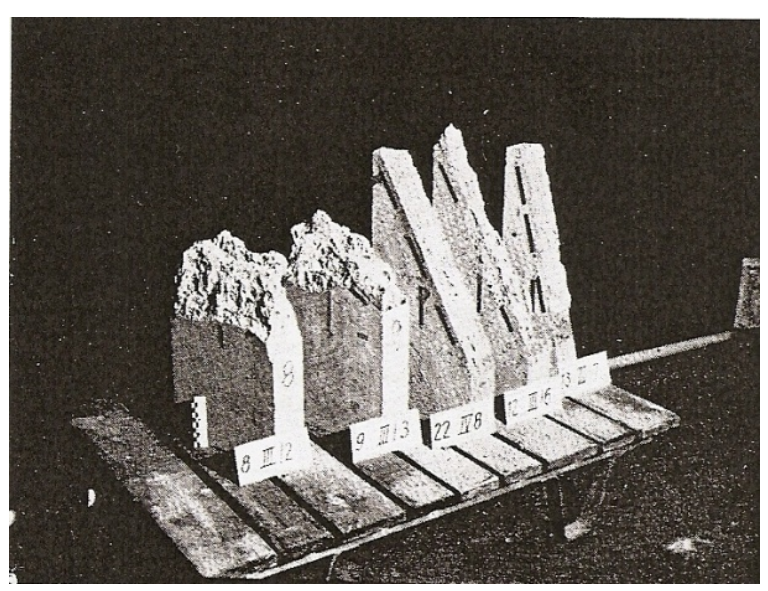

b)

Figura 2: EIBL et al. [8]: a) Esquema de ensaio; b) corpos de prova ensaiados.

KUPFER [11], a pedido do consórcio responsável pela obra, desenvolveu um trabalho para a Ponte Rio-Niterói (Ponte Presidente Costa e Silva), que avaliou o comportamento da junção de peças pré-moldadas de concreto armado por meio de ensaios (Figura 3). Esses ensaios não eram realizados no Brasil à época, daí a consulta a pesquisadores alemães. Os resultados obtidos permitiram a execução, com êxito, das aduelas prémoldadas protendidas da ponte Rio-Niterói.

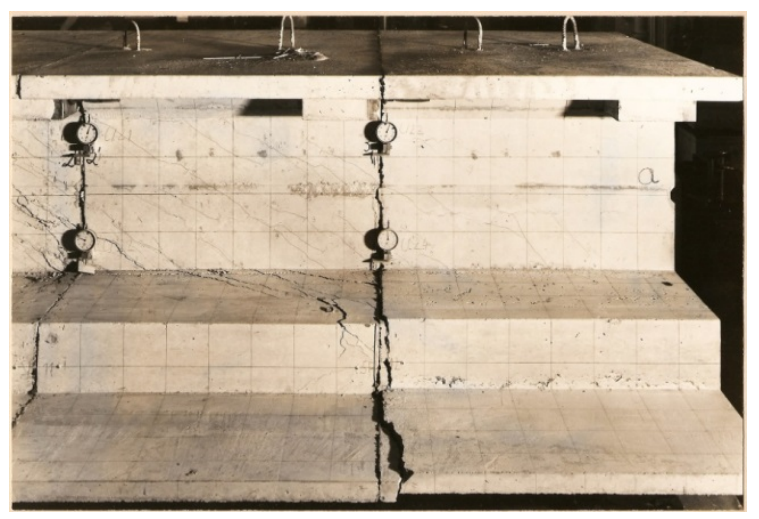

Figura 3: Ensaio realizado por KUPFER (1972).

Diversos pesquisadores contribuíram para o desenvolvimento da tecnologia que envolve juntas de concreto. MATTOCK et al. [16], MATTOCK et al. [13], MATTOCK et al. [14], REINHARDT [18], MATTOCK et al. [15], WALRAVEN e STROBAND [17], JANSZE e WALRAVEN [10], ZILCH e REINECKE [19] foram os principais pesquisadores que se dedicaram a esse assunto.

MAEKAWA et al. [12] buscaram um modelo constitutivo considerando, essencialmente, as características microfísicas da transferência de tensões, incluindo o comportamento elasto-plástico, a dilatância devido ao cisalhamento, a taxa de transferência de tensões e a geometria da superfície fissurada, propondo um modelo mais geral (Figura 4). 


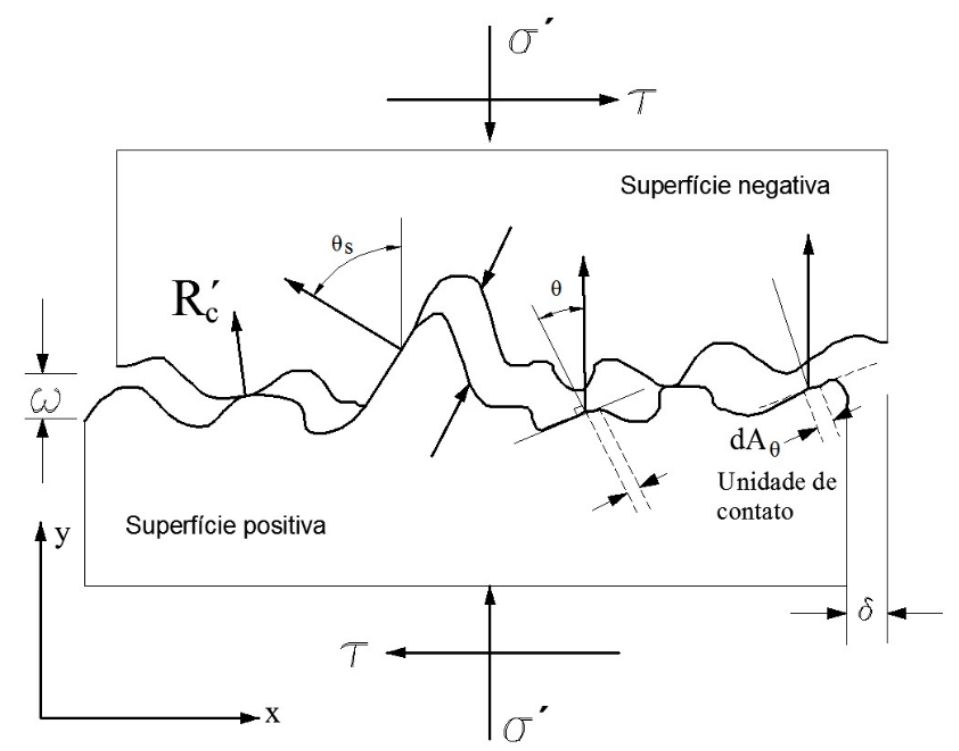

Figura 4: Esquema do modelo de MAEKAWA et al. [12].

\section{PROGRAMA EXPERIMENTAL}

No programa experimental desenvolvido por GUERRANTE [9], inicialmente foram concretados os corpos de prova cilíndricos nas resistências almejadas de $20 \mathrm{MPa}, 30 \mathrm{MPa}, 50 \mathrm{MPa}$ e $70 \mathrm{MPa}$. Esses cilindros iniciais foram cortados com inclinações de $30^{\circ}, 45^{\circ}$ e $60^{\circ}$ (Figura 5), que receberam o mesmo procedimento de corte, regularização com lixamento superficial e jateamento de água pressurizada (Figura 6).

As partes dos corpos de prova isoladas (antes da colagem) foram denominadas seguindo a nomenclatura $\mathrm{C} f c-\mathrm{A}-\mathrm{N}$, onde $\mathrm{C}$ indica cilindro, $f c$ indica a resistência média à compressão do elemento prémoldado, A é o ângulo de inclinação da junta, e N é o número de ordem do corpo de prova (Figura 6 e 7). Os corpos de prova foram executados segundo as normas ABNT NBR 5738[1] e NBR 6118[4].

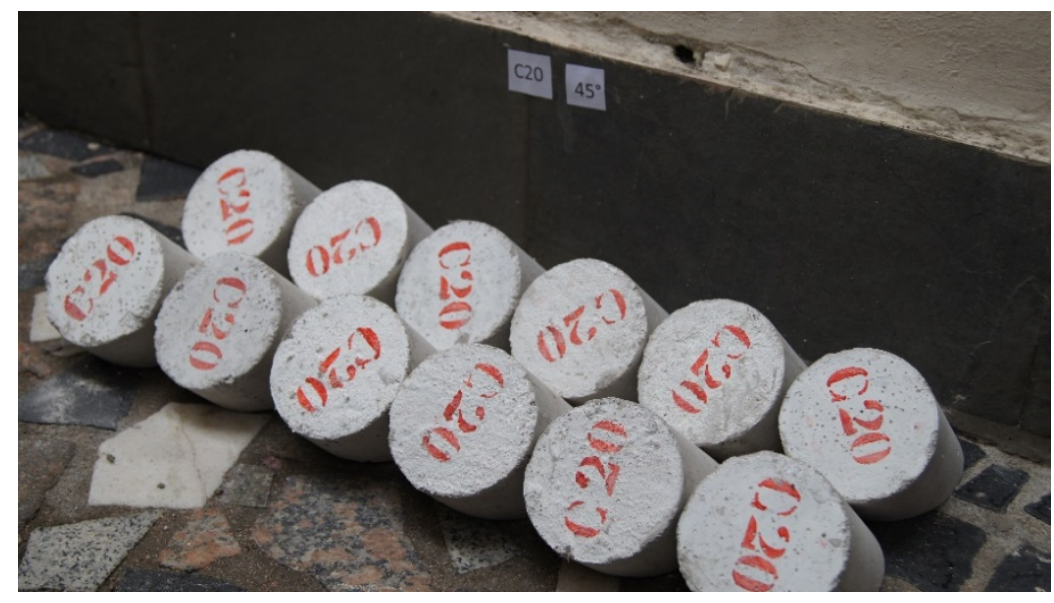

Figura 5: Vista inferior com identificação dos corpos de prova com resistência almejada de $20 \mathrm{MPa}$. 


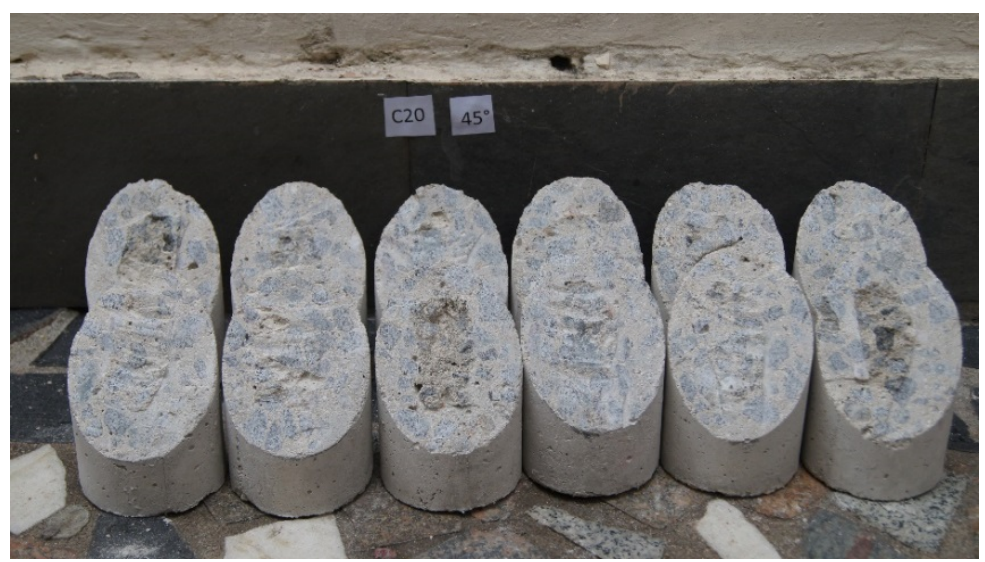

Figura 6: Vista superior dos corpos de prova com resistência almejada de $20 \mathrm{MPa}$ após o corte.

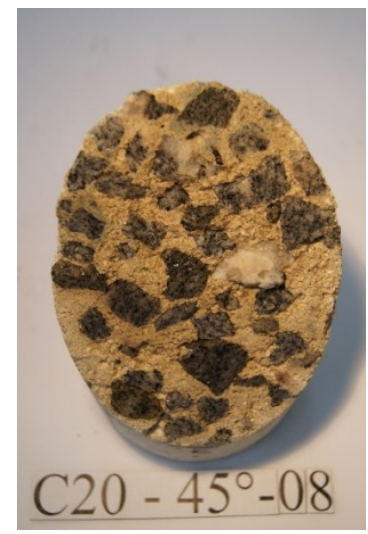

Figura 7: Vista superior do Corpo de prova $\mathrm{C} 20-45^{\circ}-08 \mathrm{MPa}$ após o corte, regularização e jateamento de água pressurizada por cerca de 15 minutos.

As características principais do adesivo epóxi utilizado são: bicomponente; fluído/pastoso; mistura A + B cinza; massa específica 1,6 a 1,8 g/cm3; consumo 0,8 a $1,0 \mathrm{~kg} / \mathrm{m} 2$, dependendo da rugosidade do substrato; tempo de trabalhabilidade de 60 minutos; resistência à compressão: $60 \mathrm{MPa}$ aos 28 dias.

Após a colagem foram montados 93 corpos de prova cilíndricos de $100 \mathrm{~mm}$ x $200 \mathrm{~mm}$, de acordo com a nomenclatura $\mathrm{C} f_{\mathrm{cS}}-f_{\mathrm{cl}}-\mathrm{A}-\mathrm{N}$, onde $\mathrm{C}$ indica cilindro, $f_{\mathrm{cS}}$ indica a resistência média à compressão da parte superior do corpo de prova, e $f_{\mathrm{cI}}$ indica a resistência média à compressão da parte inferior do corpo de prova. A é o ângulo de inclinação da junta, e $\mathrm{N}$ é o número de ordem do corpo de prova. As composições dos corpos de prova para as séries com $30^{\circ}, 45^{\circ}$ e $60^{\circ}$ são apresentadas nas Figuras 8,9 e 10.

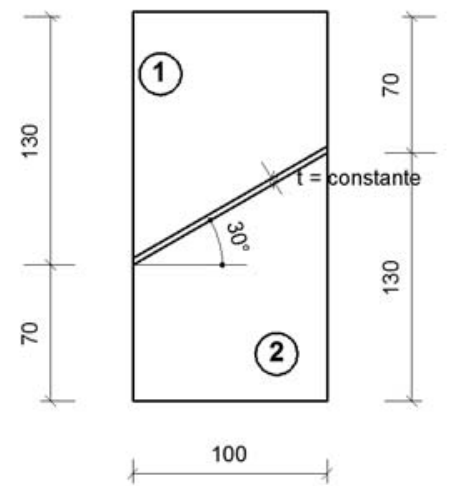

(a)
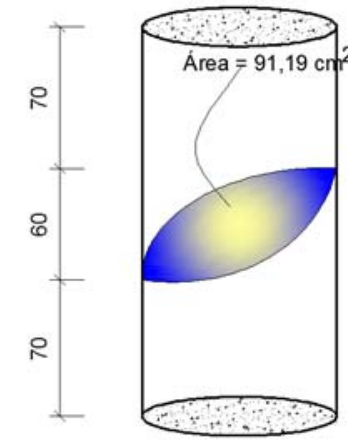

100

(b) 
Figura 8: Corpo de prova com união a $30^{\circ}$ : a) seção transversal; b) dimensões (medidas em mm).

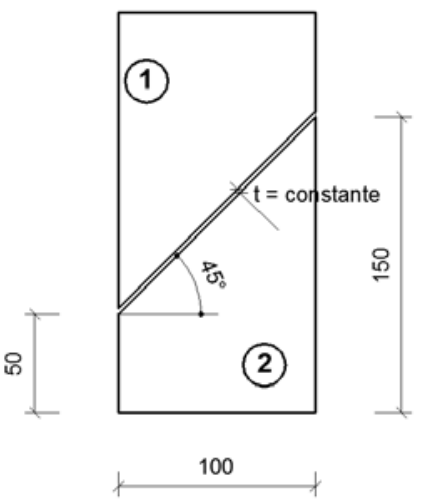

(a)

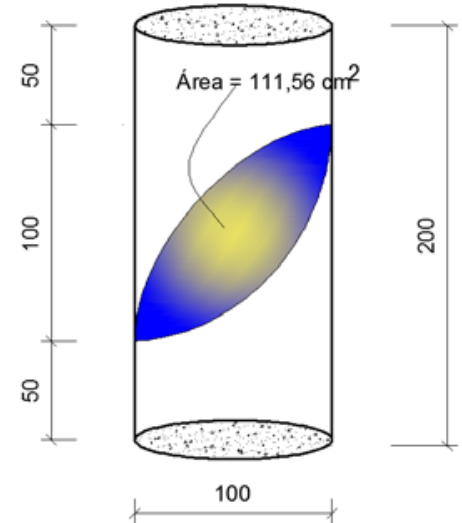

(b)

Figura 9: Corpo de prova com união a $45^{\circ}$ : a) seção transversal; b) dimensões (medidas em mm).

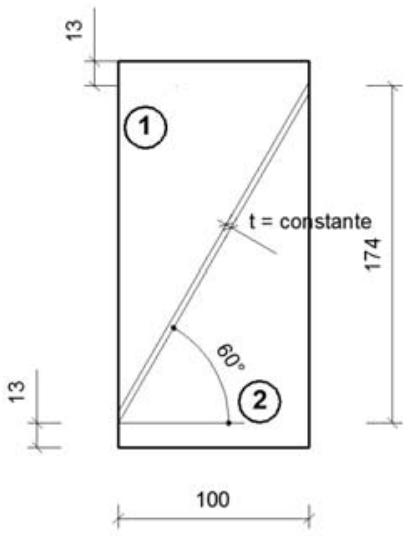

(a)

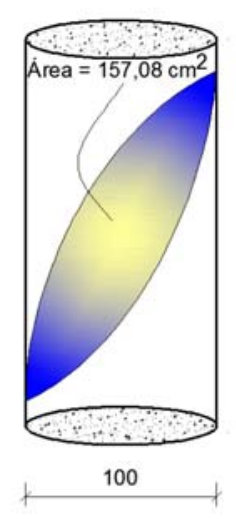

(b)

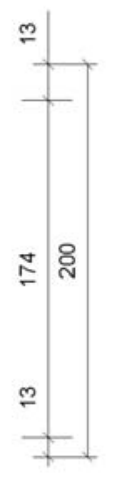

Figura 10: Corpo de prova com união a $60^{\circ}$ : a) seção transversal; b) dimensões (medidas em mm).

As séries ensaiadas foram C20S-20I, C20S-30I, C30S-30I, C30S-50I, C50S-50I, C50S-70I e C70S70I. As quantidades de ensaios realizados por série são apresentadas na Tabela 1.

Tabela 1: Número de ensaios realizados por série.

\begin{tabular}{c|c|c|c}
\hline \multirow{2}{*}{ Séries } & \multicolumn{3}{|c}{ Corpos de prova } \\
\cline { 2 - 4 } & $30^{\circ}$ & $45^{\circ}$ & $60^{\circ}$ \\
\hline C20S-20I & 4 & 5 & 4 \\
C20S-30I & 4 & 4 & 4 \\
C30S-30I & 4 & 4 & 4 \\
C30S-50I & 4 & 4 & 4 \\
C50S-50I & 4 & 4 & 6 \\
C50S-70I & 4 & 4 & 4 \\
C70S-70I & 6 & 6 & 6 \\
\hline
\end{tabular}

\subsection{Instrumentação}

Para aquisição de dados foram utilizados extensômetros elétricos de resistência (EER) do tipo PA-06201BA-120L, com comprimento de grade de 51,1 mm, para montagem das rosetas. As duas rosetas, uma em cada face das metades, foram coladas diametralmente opostas (Figura 11). 
Os ensaios foram realizados no Laboratório de Materiais de Construção e Concreto do Instituto Militar de Engenharia. A prensa tinha capacidade de carga de $1000 \mathrm{kN}$.

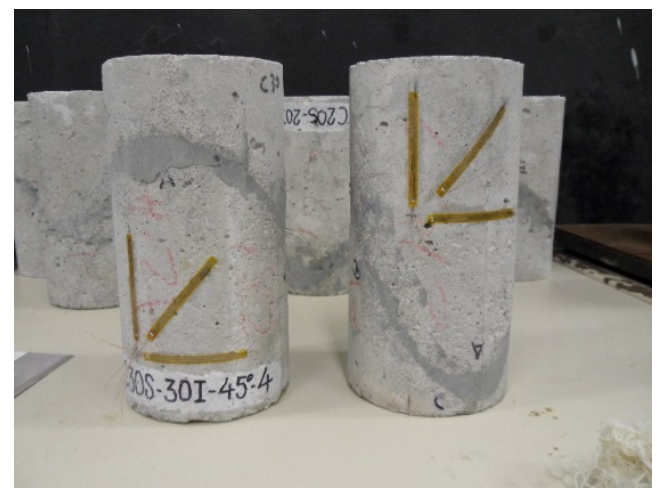

Figura 11: Instrumentação dos corpos de prova com extensômetros elétricos de resistência (EER).

\subsection{Rugosidade das superfícies}

Antes de serem coladas as superfícies dos corpos de prova foram lixadas mecanicamente e receberam jato de água pressurizada com equipamento da marca NORDTECH TEKNA, modelo HLX150V, pressão 2.175 lbs, com distância média do jato de $20 \mathrm{~cm}$ por cerca de 15 minutos para lhes dar rugosidade. Foram fotografadas por meio de microscópio digital USB da marca VEHO, modelo VMS-004, com ampliação de 20X em quatro regiões (Figura 12) cada metade, totalizando 744 fotografias.

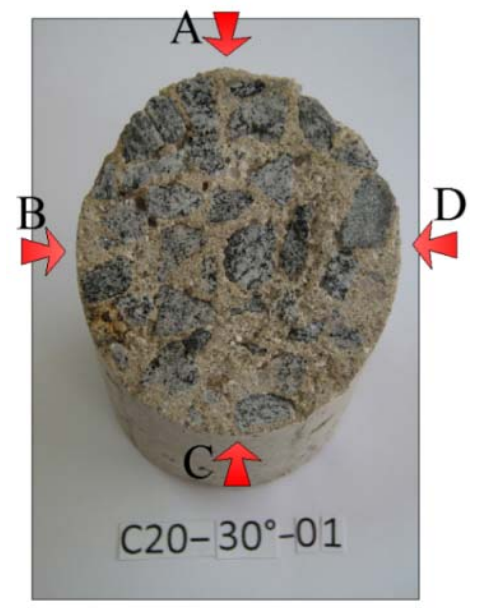

Figura 12: Regiões em cada corpo de prova cortado.

As fotografias foram obtidas com contraste por meio luz negra e traçados o relevo lateral pelo programa AutoCad (Figura 13).

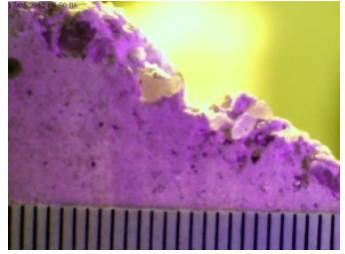

(a)

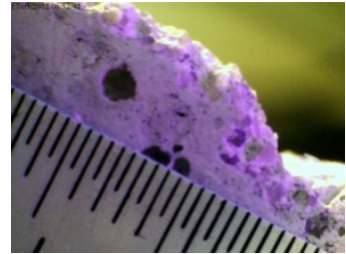

(b)

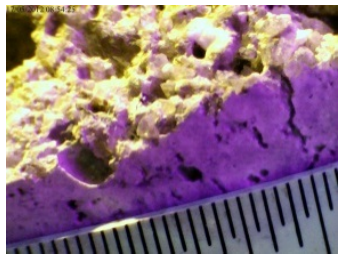

(c)

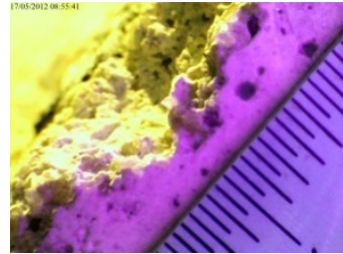

(d) 


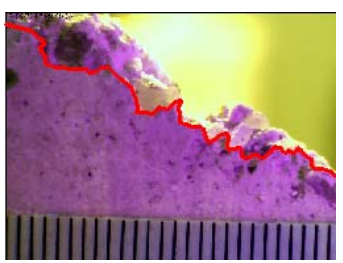

(e)

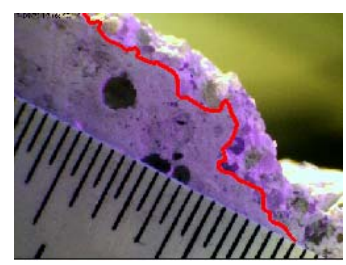

(f)

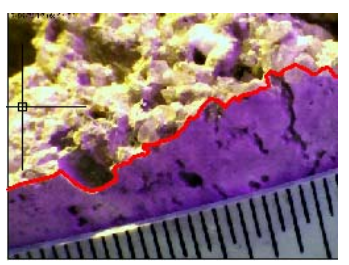

(g)

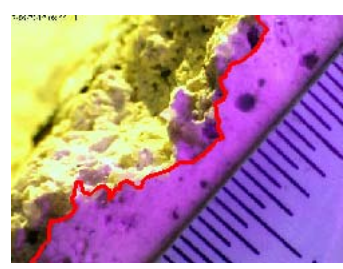

(h)

Figura 13: Ampliação de 20 vezes sem contorno: (a) C20-45-02-A; (b) C20-45-02-B; (c) C20-45-02-C; (d) C20-45-02D. ade 20 vezes com contorno: (e) C20-45-02-A; (f) C20-45-02-B; (g) C20-45-02-C e (h) C20-45-02-D.

Esses perfis foram rotacionados para a horizontal e montado um perfil composto para cada corpo de prova a ser unido (Figura 14), totalizando 186 perfis compostos. Em função desses contornos as cotas das ordenadas de cada ponto da curva foram tratadas pelas diferenças relativas entre o ponto de ordenada máxima e os demais pontos, e foram listadas todas as diferenças de altura entre os contornos. As alturas relativas possibilitaram, por meio de uma função densidade, estimar a rugosidade média aplicada. A Figura 15 representa a distribuição normal e a distribuição normal padronizada para o corpo de prova C20-30-01. Foram elaboradas 186 funções relativas a cada metade do corpo de prova.

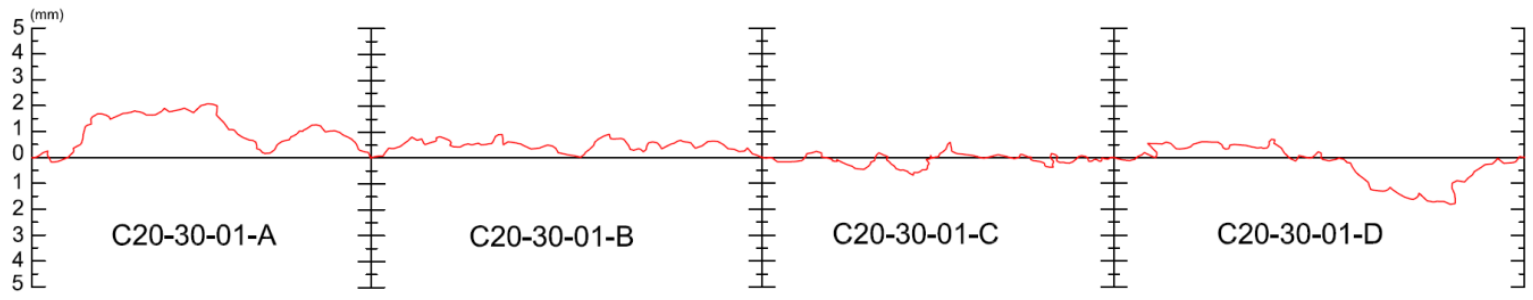

Figura 14: Junção dos contornos das faces do corpo de prova C20-30-01.

C20-30-01

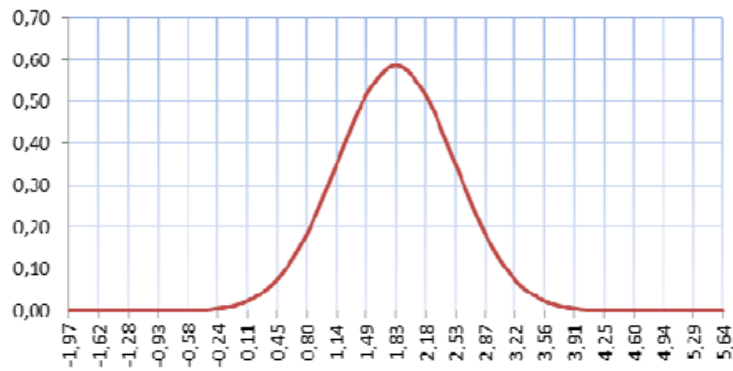

(a)
C20-30-01 PAD

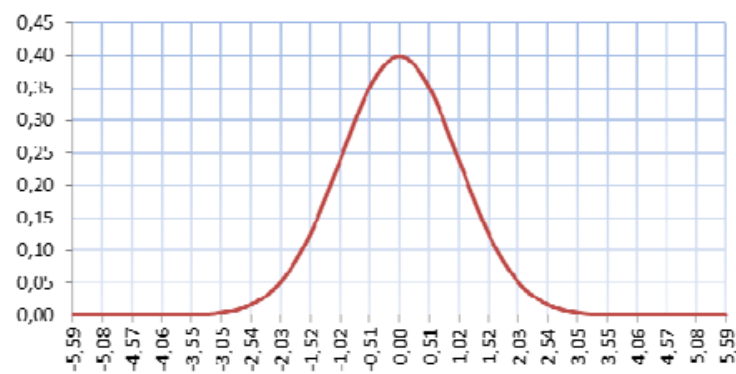

(b)

Figura 15: Distribuições normais para C20-30-01: (a) rugosidade em mm; (b) normalizada.

As rugosidades medidas em todos os corpos de prova foram analisadas para todas as classes de concreto, determinando-se a maior frequência para cada classe, tendo-se como critério o espectro de frequência (Eq.01) (ZILL e WRIGTH [20]). Os valores médios e frequências para as classes de concreto são apresentados na Tabela 2. Assim:

$$
\left|c_{n}\right|=\frac{\operatorname{senh} \pi}{\pi} \cdot \frac{1}{\sqrt{n^{2}+1}}
$$


onde

$c_{n}-$ coeficiente de frequência;

$n$ - valor da rugosidade no ponto.

As expressões obtidas para as rugosidades médias dos corpos de prova são:

- séries C21,6

$r_{21,6}=2,013+0,000293 \cos (\omega x)+0,63158 \operatorname{sen}(\omega x)$

- séries C30,0

$r_{30,0}=1,354-0,00112 \cos (\omega x)+0,32669 \operatorname{sen}(\omega x)$

- séries $\mathrm{C} 72,8$

$r_{72,8}=1,230-0,00105 \cos (\omega x)+0,24622 \operatorname{sen}(\omega x)$

- séries C86,6

$r_{96,6}=1,067-0,00181 \cos (\omega x)+0,19771 \operatorname{sen}(\omega x)$

sendo $x$ o comprimento do trecho no qual é medido a rugosidade, em $1 \mathrm{~mm}$, e os valores do parâmetro $\omega$ são apresentados na Tabela 2.

Tabela 2: Rugosidade média e maior frequência das classes de concreto.

\begin{tabular}{c|c|c}
\hline Classes & Rugosidade média $(\mathbf{m m})$ & Frequência $(\boldsymbol{\omega})$ \\
\hline C21,6 & 2,013 & 2,04 \\
C30,0 & 1,354 & 2,08 \\
C72,8 & 1,230 & 2,22 \\
C 86,6 & 1,067 & 2,50 \\
\hline
\end{tabular}

As representações gráficas dessas expressões são mostradas na Figura 16, observando-se diferenças fundamentais entre os concretos de menor resistência e os de alta resistência.

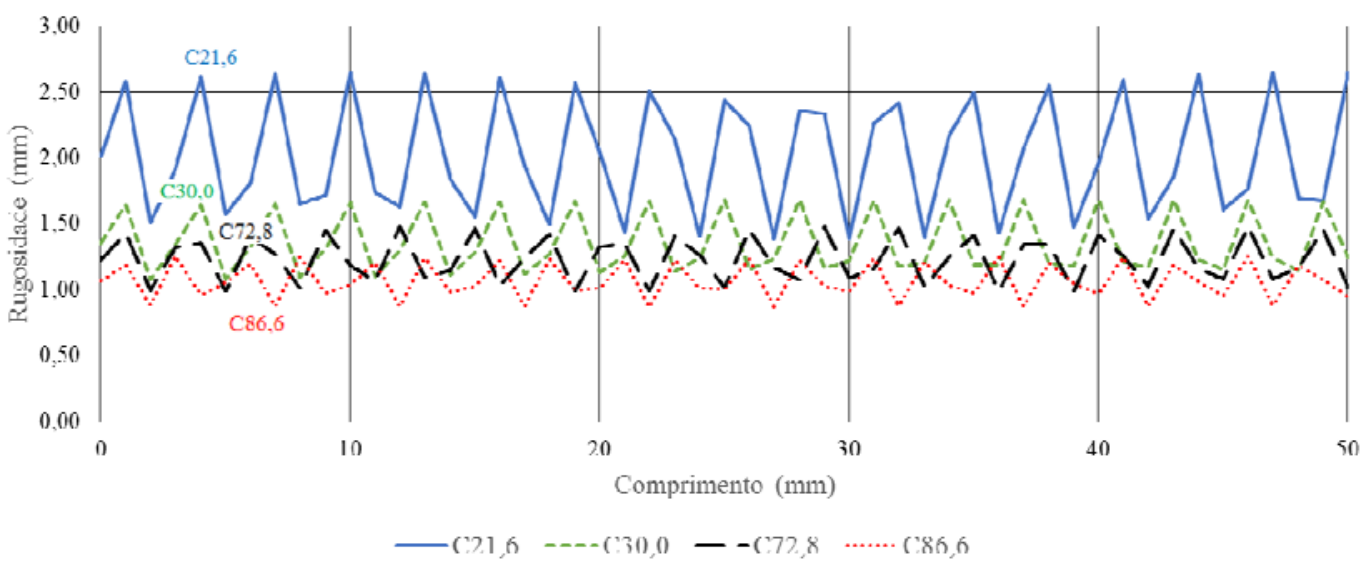

Figura 16: Rugosidades médias para os corpos de prova C21,6, C30,0, C72,8 e C86,6. 


\section{ANÁLISE DOS RESULTADOS}

As tensões normais e tangenciais aos planos considerados foram calculadas com o uso das expressões 4.5 e 4.6 de acordo com as representações da Figura 11.

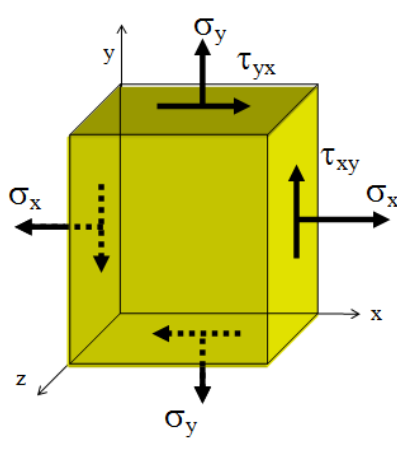

(a)

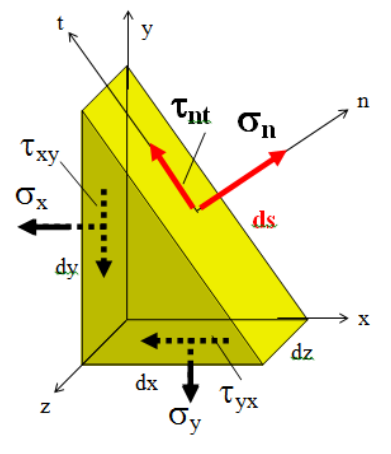

(b)

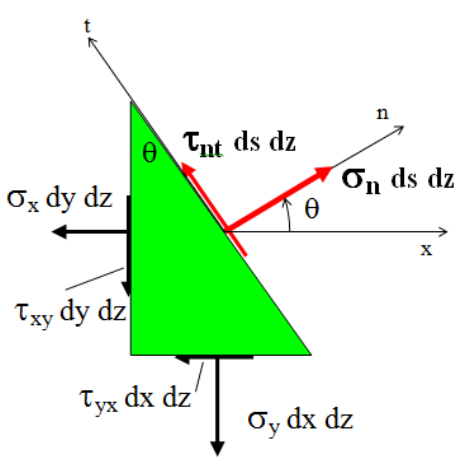

(c)

Figura 17: Variação da tensão com a orientação do plano da seção: (a) estado duplo de tensões;

(b) tensões num plano inclinado; (c) forças atuando num plano inclinado.

Nesse caso o estado de tensão fica definido pela matriz:

$\sigma_{i j}=\left[\begin{array}{cc}\sigma_{x} & \tau_{x y} \\ \tau_{y x} & \sigma_{y y}\end{array}\right]$

Seguindo-se para o plano inclinado com ângulo $\theta$ :

$$
\begin{aligned}
& \sigma_{n}=\sigma_{x} \cos ^{2} \theta+\sigma_{y} \operatorname{sen}^{2} \theta+2 \tau_{x y} \operatorname{sen} \theta \cos \theta \\
& \tau_{n t}=-\left(\sigma_{x}-\sigma_{y}\right) \operatorname{sen} \theta \cos \theta+\tau_{x y}\left(\cos ^{2} \theta-\operatorname{sen}^{2} \theta\right)
\end{aligned}
$$

As Tabelas 3, 4 e 5 apresentam os resultados médios válidos para tensão de ruptura, tensão normal ao plano e tensão de aderência para inclinações de $30^{\circ}, 45^{\circ}$ e $60^{\circ}$, respectivamente.

\begin{tabular}{|c|c|c|c|c|c|c|c|c|c|}
\hline \multirow[b]{2}{*}{ Séries } & \multicolumn{3}{|c|}{$30^{\circ}$} & \multicolumn{3}{|c|}{$45^{\circ}$} & \multicolumn{3}{|c|}{$60^{\circ}$} \\
\hline & $\begin{array}{c}f_{\mathrm{c} 30} \\
(\mathrm{MPa})\end{array}$ & $\begin{array}{c}\mathrm{DP} \\
(\mathrm{MPa})\end{array}$ & $\begin{array}{l}\text { CV } \\
\text { (\%) }\end{array}$ & $\begin{array}{c}f_{c 45} \\
(\mathrm{MPa})\end{array}$ & $\begin{array}{c}\mathrm{DP} \\
(\mathrm{MPa})\end{array}$ & $\begin{array}{l}\text { CV } \\
(\%)\end{array}$ & $\begin{array}{c}f_{\mathrm{c} 60} \\
(\mathrm{MPa})\end{array}$ & $\begin{array}{c}\mathrm{DP} \\
(\mathrm{MPa})\end{array}$ & $\begin{array}{l}\text { CV } \\
\text { (\%) }\end{array}$ \\
\hline C20S-20I & 19,20 & 0,52 & 2,69 & 19,49 & 0,50 & 2,55 & 17,28 & 3,09 & 17,88 \\
\hline C20S-30I & 20,61 & 1,81 & 8,78 & 18,00 & 0,82 & 4,58 & 20,58 & 0,04 & 0,21 \\
\hline C30S-30I & 25,43 & 0,62 & 2,44 & 26,44 & 0,09 & 0,32 & 22,49 & 0,62 & 2,76 \\
\hline C30S-50I & 32,46 & 0,37 & 1,15 & 26,23 & 3,02 & 11,52 & 35,12 & 2,86 & 8,13 \\
\hline C50S-50I & 57,37 & 1,11 & 1,93 & 49,08 & 2,74 & 5,58 & 33,07 & 10,69 & 32,32 \\
\hline C50S-70I & 49,82 & 10,64 & 21,63 & 52,47 & 2,51 & 4,79 & 45,80 & 5,31 & 11,60 \\
\hline C70S-70I & 60,60 & 2,61 & 4,31 & 46,93 & 2,35 & 5,00 & 38,18 & 4,91 & 12,86 \\
\hline
\end{tabular}

Tabela 3: Valores médios para tensão de ruptura. 
Tabela 4: Valores médios para tensão normal ao plano inclinado.

\begin{tabular}{c|c|c|c|c|c|c|c|c|c}
\hline \multirow{2}{*}{ Séries } & \multicolumn{3}{|c|}{$\mathbf{3 0}^{\circ}$} & \multicolumn{3}{c|}{$\mathbf{4 5}^{\circ}$} & \multicolumn{3}{c}{$\mathbf{6 0}^{\circ}$} \\
\cline { 2 - 11 } & $\sigma_{N 30}$ & DP & $\mathbf{C V}$ & $\sigma_{N 45}$ & DP & $\mathbf{C V}$ & $\sigma_{N 60}$ & DP & CV \\
& $(\mathbf{M P a})$ & $\mathbf{M P a})$ & $\mathbf{( \% )}$ & $\mathbf{( M P a )}$ & $\mathbf{( M P a )}$ & $\mathbf{( \% )}$ & $(\mathbf{M P a})$ & $\mathbf{( M P a )}$ & $\mathbf{( \% )}$ \\
\hline C20S-20I & 14,40 & 0,39 & 2,70 & 9,75 & 0,24 & 2,51 & 4,32 & 0,16 & 3,77 \\
C20S-30I & 15,46 & 1,36 & 8,78 & 9,00 & 0,41 & 4,55 & 5,15 & 0,01 & 0,14 \\
C30S-30I & 19,07 & 0,47 & 2,45 & 13,22 & 0,05 & 0,34 & 5,62 & 0,15 & 2,67 \\
C30S-50I & 24,34 & 0,28 & 1,16 & 13,12 & 1,51 & 11,51 & 8,78 & 0,71 & 8,12 \\
C50S-50I & 43,03 & 0,83 & 1,94 & 24,54 & 1,36 & 5,58 & 8,26 & 2,67 & 32,31 \\
C50S-70I & 37,36 & 7,98 & 21,35 & 26,24 & 1,26 & 4,79 & 10,39 & 2,38 & 22,88 \\
C70S-70I & 45,45 & 1,96 & 4,31 & 23,47 & 1,17 & 4,99 & 9,54 & 1,23 & 12,86 \\
\hline
\end{tabular}

Tabela 5: Valores médios para tensão de aderência.

\begin{tabular}{c|c|c|c|c|c|c|c|c|c}
\hline \multirow{2}{*}{ Séries } & \multicolumn{3}{|c|}{$\mathbf{3 0}^{\circ}$} & \multicolumn{3}{c|}{$\mathbf{4 5}^{\circ}$} & \multicolumn{3}{c}{$\mathbf{6 0}^{\circ}$} \\
\cline { 2 - 10 } & $\boldsymbol{\tau}_{A 30}$ & $\mathbf{D P}$ & $\mathbf{C V}$ & $\boldsymbol{\tau}_{A 45}$ & $\mathbf{D P}$ & $\mathbf{C V}$ & $\boldsymbol{\tau}_{A 60}$ & $\mathbf{D P}$ & $\mathbf{C V}$ \\
& $\mathbf{M P a})$ & $\mathbf{M P a})$ & $\mathbf{( \% )}$ & $\mathbf{M P a})$ & $\mathbf{M P a}$ & $\mathbf{( \% )}$ & $\mathbf{( M P a )}$ & $\mathbf{( M P a )}$ & $\mathbf{( \% )}$ \\
\hline C20S-20I & 8,31 & 0,22 & 2,72 & 9,75 & 0,24 & 2,51 & 7,48 & 0,28 & 3,78 \\
C20S-30I & 8,93 & 0,78 & 8,79 & 9,00 & 0,41 & 4,55 & 8,92 & 0,02 & 0,24 \\
C30S-30I & 11,01 & 0,27 & 2,41 & 13,22 & 0,05 & 0,34 & 9,74 & 0,27 & 2,72 \\
C30S-50I & 14,06 & 0,16 & 1,16 & 13,12 & 1,51 & 11,51 & 15,21 & 1,24 & 8,14 \\
C50S-50I & 24,84 & 0,48 & 1,94 & 24,54 & 1,37 & 5,58 & 14,32 & 4,63 & 32,32 \\
C50S-70I & 21,57 & 4,61 & 21,38 & 26,24 & 1,26 & 4,79 & 19,53 & 2,30 & 11,60 \\
C70S-70I & 26,24 & 1,13 & 4,30 & 23,47 & 1,17 & 4,99 & 16,53 & 2,13 & 12,86 \\
\hline
\end{tabular}

\subsection{Ruptura dos corpos de prova}

Os ensaios foram realizados conforme as normas ABNT NBR 5379 [2], NBR 7222 [3] e NBR 8522 [5].

Os corpos de prova de concreto de resistência normal apresentaram ruptura monolítica independente da inclinação da junta. Os corpos de prova de concreto de alta resistência e inclinação de $30^{\circ}$ tiveram o comportamento monolítico na ruptura, e todos os de concreto de alta resistência e inclinações de junta de $45^{\circ}$ e $60^{\circ}$ apresentaram rompimento na junta de colagem (Figura 18).

Na Tabela 6 são apresentados os tipos de rupturas ocorridas nas séries ensaiadas.

Tabela 6: Tipos de ruptura em função da inclinação da junta.

\begin{tabular}{c|c|c|c}
\hline \multirow{2}{*}{ Série } & \multicolumn{3}{|c}{ Inclinação da junta } \\
\cline { 2 - 4 } & $\mathbf{3 0}^{\mathbf{0}}$ & $\mathbf{4 5}^{\mathbf{0}}$ & $\mathbf{6 0}^{\mathbf{0}}$ \\
\hline C20S-20I & monolítica & monolítica & monolítica \\
C20S-30I & monolítica & monolítica & monolítica \\
C30S-30I & monolítica & monolítica & monolítica \\
C30S-50I & monolítica & Junta de colagem & Junta de colagem \\
C50S-50I & monolítica & Junta de colagem & Junta de colagem \\
C50S-70I & monolítica & Junta de colagem & Junta de colagem \\
C70S-70I & monolítica & & \\
\hline
\end{tabular}




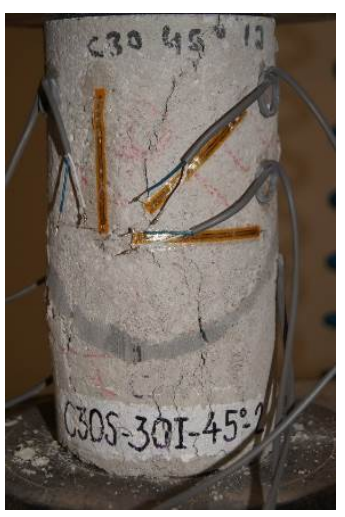

(a)

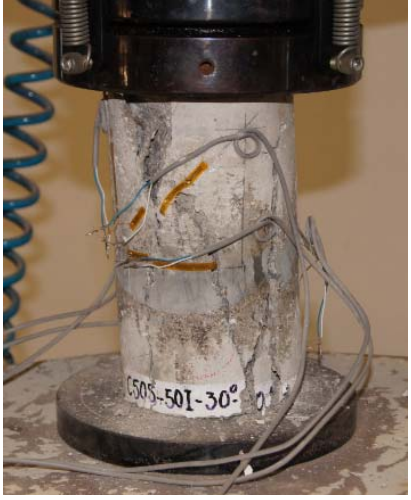

(b)

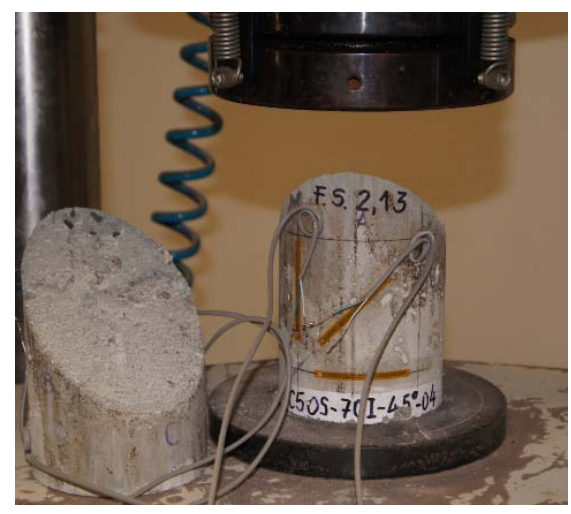

(c)

Figura 18: Rupturas dos corpos de prova: (a) comportamento monolítico na ruptura para corpos de prova de concreto de resistência normal e qualquer inclinação; (b) comportamento monolítico na ruptura para corpos de prova de concreto de alta resistência e inclinação de $30^{\circ}$; (c) comportamento monolítico na ruptura para corpos de prova de concreto de alta resistência e inclinações de $45^{\circ}$ e $60^{\circ}$.

\subsection{Relação entre rugosidade média e tensão de aderência}

As variações da rugosidade média das classes de concreto podem ser relacionadas ao comportamento da tensão de aderência. Essas relações, que são inversamente proporcionais, estão apresentadas de forma normalizada nas Figuras 19 e 20 para as séries de concreto de resistência normal (CRN) e de alta resistência (CAR), respectivamente. Nessas Figuras foram utilizadas as relações entre os valores de rugosidade média e tensão de aderência e os valores máximos obtidos, respectivamente, para cada série pesquisada.

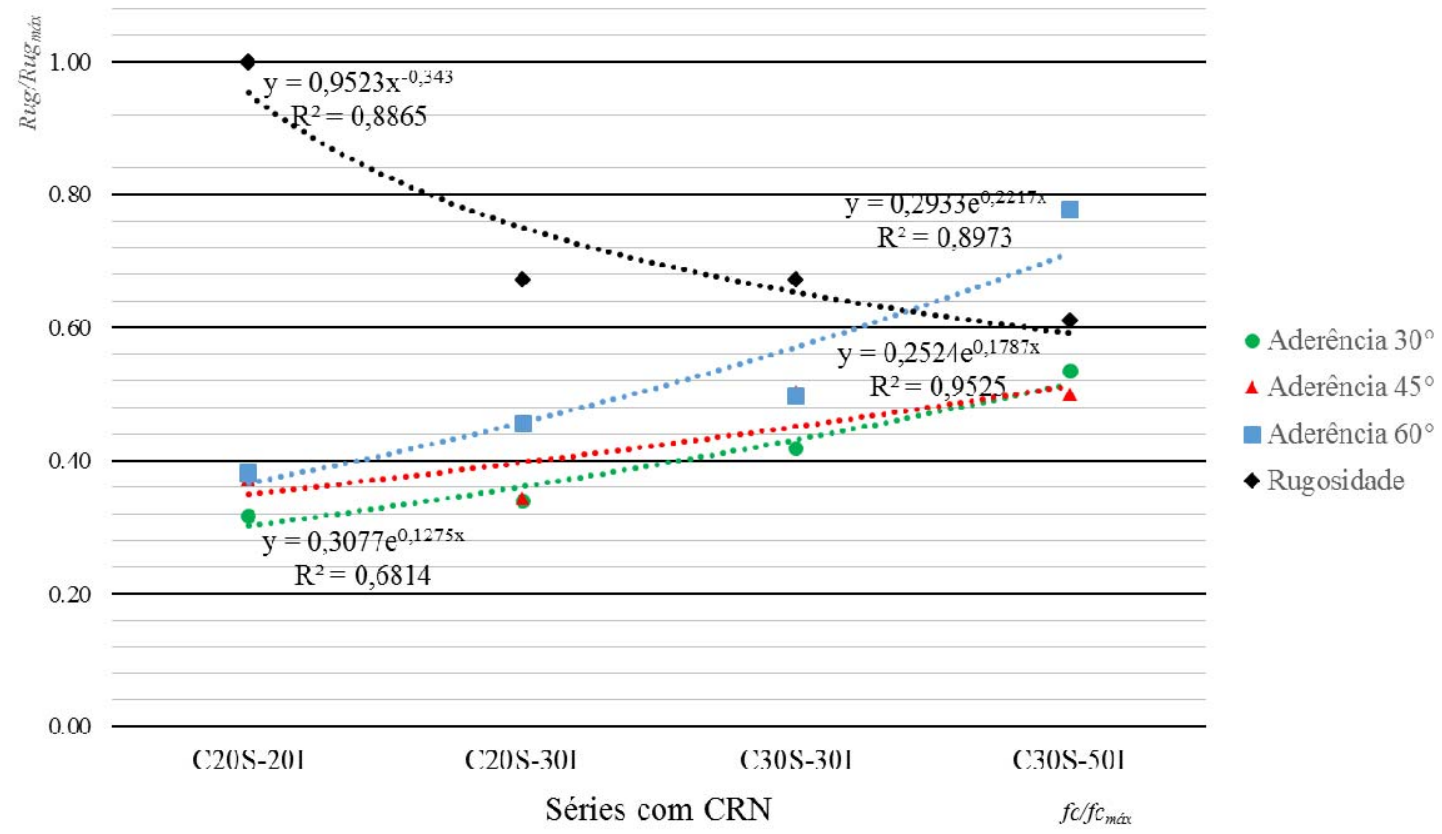

Figura 19: Rugosidade média vs aderência para CRN, para cada série normalizada. 


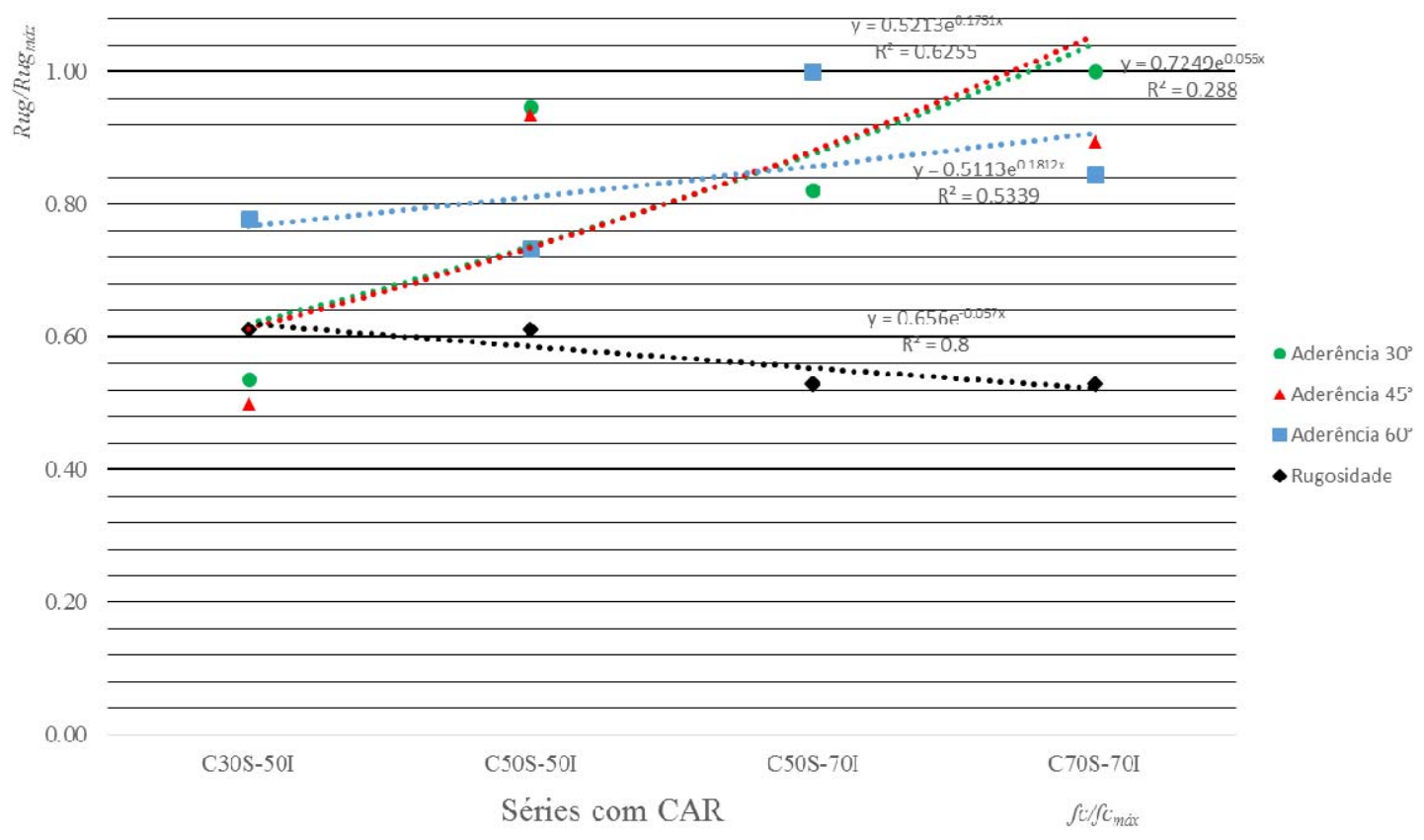

Figura 20: Rugosidade média vs aderência para CAR, para cada série normalizada.

\subsection{Comportamento da tensão de aderência}

As tensões de aderência para os corpos de prova que tiveram ruptura monolítica (C20S-20I, C20S-30I, C30S30I e C30S-50I) foram calculadas com o uso das expressões 7 e 8.

Nenhum corpo de prova com concreto de menor resistência (CRN) na parte superior rompeu na região da junta, que é o caso das séries C20S-20I, C20S-30I, C30S-30I e C30S-50I.

As tensões de aderência das séries C50S-50I, C50S-70I e C70S-70I com inclinação da junta de $30^{\circ}$ também tiveram seus valores calculados por meio das expressões 7 e 8 . As séries com $30^{\circ}$ tiveram valores médios 5,2\% maiores que os das séries com ângulo de $45^{\circ}$ e $51,3 \%$ maiores que os da série de $60^{\circ}$. Essas duas últimas séries romperam todas pelas juntas de colagem.

A tensão de aderência para as séries C20S-20I, C20S-30I, C30S-30I e C30S-50I apresentou uma evolução linear e diretamente proporcional ao aumento da resistência, com pouca influência do ângulo de inclinação da junta.

A utilização de concretos de alta resistência mostrou que a tensão de aderência dessas séries alcançou valores inversamente proporcionais ao aumento do ângulo de inclinação.

O comportamento da tensão de aderência das séries ensaiadas é mostrado na Figura 21. 


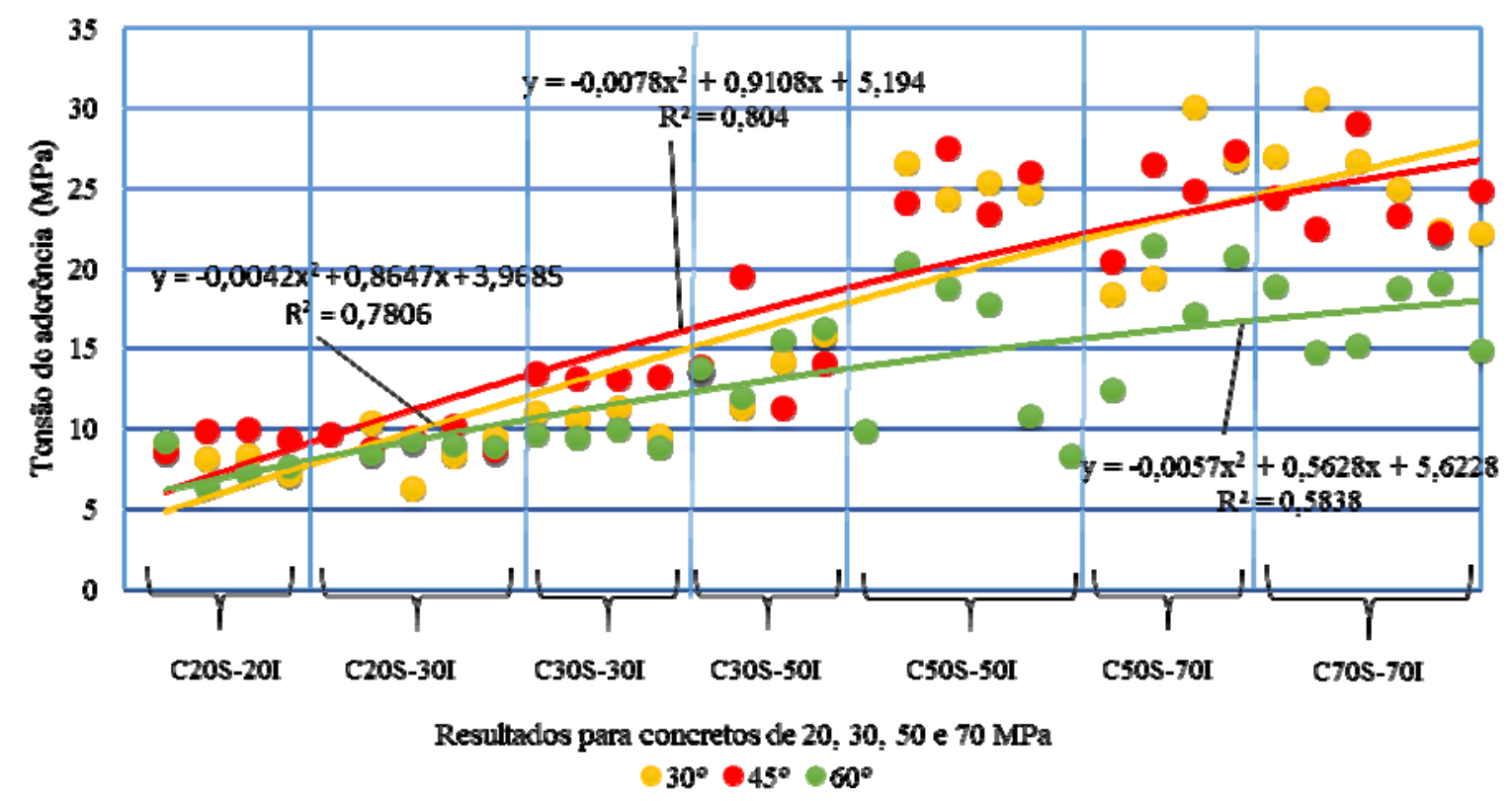

Figura 21: Evolução da tensão de aderência em função do aumento da resistência do concreto.

\section{CONCLUSÕES}

O aumento da espessura de adesivo epóxi, em razão de deficiências no plano de colagem, a menor rugosidade média e a resistência à compressão do adesivo provocaram variações mais evidentes nas tensões dos corpos de prova compostos por concreto de alta resistência.

Todos os corpos de prova de CRN apresentaram comportamento monolítico na ruptura. A série C30S50I apresentou rompimento monolítico com ocorrência de fendilhamentos principalmente na metade superior (o de menor resistência C30).

Os resultados obtidos nos ensaios de corpos de prova formados por CRN corroboram os obtidos nas pesquisas de JANSZE e WALRAVEN [10], que relataram a ruptura no concreto e não na junta de colagem.

Os corpos de prova de CAR e com inclinação da junta de $30^{\circ}$ apresentaram a ruptura de forma monolítica (Figura 18.(b)). Todas as séries de CAR e inclinação de $45^{\circ}$ e $60^{\circ}$ (C50S-50I-45, C50S-70I-45, C70S-7OI-45, C50S-50I-60, C50S-70I-60, C70S-7OI-60) apresentaram a ruptura de forma brusca na junta de colagem.

Os resultados obtidos comprovaram que o aumento da resistência do CAR eleva o risco de ruptura na junta de colagem de forma brusca, como relataram JANSZE e WALRAVEN [10] (Figura 18 (c)).

Para os valores médios da tensão de ruptura, verificou-se a predominância de três tipos de ocorrências para $\alpha=30^{\circ}$, três maiores ocorrências para $\alpha=45^{\circ}$ e uma maior ocorrência para $\alpha=60^{\circ}$ (Tabela 3 ).

Em relação aos valores médios para tensão normal ao plano inclinado, todas as maiores ocorrências foram para $\alpha=30^{\circ}$ (Tabela 4 ).

Para os valores médios para tensão de aderência, verificaram-se duas maiores ocorrências para $\alpha=$ $30^{\circ}$, quatro maiores ocorrências para $\alpha=45^{\circ}$ e uma maior ocorrência para $\alpha=60^{\circ}$ (Tabela 5).

A combinação de CRN e CAR, aliada a uma maior área de colagem $\left(\alpha=60^{\circ}\right)$, e a menor deformação na parte inferior do corpo de prova proporcionaram valores mais elevados para a tensão de ruptura e para a tensão de aderência na série C30S-50I.

Para os corpos de prova de CRN, a evolução da aderência para $\alpha=30^{\circ}$ e $\alpha=45^{\circ}$ teve praticamente o mesmo comportamento, tendo acréscimo gradativo com o aumento da resistência do concreto. Para os corpos de prova de CRN e CAR (C30S-50I) e $\alpha=60^{\circ}$, a aderência apresentou um significativo aumento em relação aos de CRN. Para os corpos de prova de CAR os comportamentos da aderência para $\alpha=30^{\circ}$ e $\alpha=45^{\circ}$ foram praticamente idênticos. Os corpos de prova de CAR apresentaram valores de aderência maiores que os da série C30S-50I e $\alpha=60^{\circ}$, mas esse comportamento não apresentou tendência de aumento acentuada como na série mencionada, mostrando para as séries C50S-50I, C50S-70I e C70S-70I uma tendência de crescimento menor.

As rugosidades médias são inversamente proporcionais à aderência em cada inclinação, sendo maior para CRN e menor para CAR. 
As variáveis mais susceptíveis no CAR, como a rugosidade média menor, a espessura e a resistência à compressão do adesivo, influenciaram os resultados da capacidade resistente desses corpos de prova.

\section{BIBLIOGRAFIA}

[1] ASSOCIAÇÃO BRASILEIRA DE NORMAS TÉCNICAS. NBR 5738 - Procedimento para moldagem e cura de corpos-de-prova - Método de ensaio. Rio de Janeiro, RJ, Brasil, dez. 2003.

[2] _. NBR 5739 - Concreto - Ensaio de compressão de corpos de prova cilíndricos - Método de ensaio. Rio de Janeiro, RJ, Brasil, mar. 2007.

[3] _ _. NBR 7222 - Determinação da resistência à tração por compressão diametral de corpos-de-prova cilíndricos - Método de ensaio. Rio de Janeiro, RJ, Brasil, mar. 1994.

[4]__. NBR 6118 - Projeto de estruturas de concreto - Procedimento, Rio de Janeiro, RJ, Brasil, abr. 2004.

[5]__. NBR 8522 - Concreto - Determinação do módulo estático de elasticidade à compressão - Método de ensaio, Rio de Janeiro, RJ, Brasil, abr. 2008.

[6] BIRKLAND, P.W.; BIRKLAND, H. W. "Connections in precast concrete construction", ACI Journal, v.63, n.3, march 1966, pp.345-368.

[7] COMITÉ EURO-INTERNATIONAL DU BÉTON. FIB BULLETIN 55: Model Code 2010. London, Thomas Telford, v.1, 2010.

[8] EIBL, J. FRANKE, L. HJORTH, O., Versuche mit kunsthärzmörteln. Die Bautechnik, Ernst \& Sohn, n.10, 1972. P.348-354.

[9] GUERRANTE, I. F. Estudo teórico e experimental da junção de concretos de diferentes resistências por meio de adesivo epóxi., Tese de D.Sc, Faculdade de Engenharia da UFF, Universidade Federal Fluminense, Rio de Janeiro, 2013.

[10] JANSZE, W., WALRAVEN, J. C. "Failure behavior of joints between HSC members, subjected to shear and compressive stresses". In: 4th International Symposium on utilizations of high strength/high performance concrete, Paris, 1996.

[11] KUPFER, H. Untersuchung des Tragverhaltens eines aus Betonfertigteilen zusammengespannten Trägers mit Verklebung in den Fugen, München, Technische Hochschule München, 1972.

[12] MAEKAWA, K.., PIMANMAS, A., OKAMURA, H. Nonlinear mechanics of reinforced concrete, USA, Spon Press, 2004.

[13] MATTOCK, A. H., CHOW, H. C., JOHAL, L. Shear transfer in reinforced concrete with moment or tension acting across the shear plane, PCI Journal, USA. July-August, 1975.

[14] MATTOCK, A. H., LI, W. K., WANG, T. C. Shear transfer in lightweight reinforced concrete, PCI Journal, Jan-Feb, 1976.

[15] MATTOCK, A. H., MAU, S. T., HSU, T. T. C. "Discussion of WALRAVEN (1987)", PCI Journal, v.33, n.1, Jan-Feb, 1988.

[16] MATTOCK, A. H., WAWKINS, N. M. Shear transfer in reinforced concrete - Recent research. PCI Journal, USA. March-April, 1972.

[17] WALRAVEN, J. C., STROBAND, J. "Shear friction in high-strength concrete". ACI-Conference, High-Strength Concrete, Singapore, 1993.

[18] REINHARDT, H. W. "Length influence on bond shear strength of joints in composite precast concrete slabs", International Journal of Cement Composites and Lightweight Concrete, v.4, n.3, August, 1982.

[19] ZILCH, K., REINECKE, R. "Capacity of shear joints between high-strength precast elements and normal cast-in-place decks". In: International Symposium on high-performance concrete - The economical solution for durable bridges and transportation structures, PCI/FHWA/FIB, Precast/Prestressed Concrete Institute, USA, Orlando, 2000.

[20] ZILL, D. G., WRIGTH, W. S. Advanced engineering mathematics., 4th ed. USA, Jones and Bartlett Publishers, 2011. 